\title{
Molecular Epidemiology and Antigenic Characterization of Seasonal Influenza Viruses Circulating in Nepal
}

\author{
Bishnu Prasad Upadhyay, ${ }^{1,2}$ Prakash Ghimire, ${ }^{2}$ Masato Tashiro, ${ }^{3}$ Megha Raj Banjara ${ }^{2}$ \\ ${ }^{1}$ National Public Health Laboratory, Department of Health Services, Kathmandu, Nepal, ${ }^{2}$ Central Department \\ of Microbiology, Tribhuvan University, Kirtipur, Nepal, ${ }^{3}$ National Institute of Infectious Diseases, Tokyo, \\ Japan.
}

\begin{abstract}
Background: Influenza is one of the public health burdens in Nepal and its epidemiology is not clearly understood. The objective of this study was to explore the molecular epidemiology and the antigenic characteristics of the circulating influenza viruses in Nepal.

Methods: A total of 1495 throat swab specimens were collected from January to December, 2014. Real time PCR assay was used for identification of influenza virus types and subtypes. Ten percent of the positive specimens were randomly selected and inoculated onto Madin-Darby Canine Kidney Epithelial cells (MDCK) for influenza virus isolation. All viruses were characterized by the hemagglutination inhibition (HI) assay.

Results: Influenza viruses were detected in $421 / 1495$ (28.2\%) specimens. Among positive cases, influenza A virus was detected in $301 / 421$ (71.5\%); of which 120 (39.9\%) were influenza A/H1N1 pdm09 and 181 (60.1\%) were influenza A/H3 subtype. Influenza B viruses were detected in 1 19/421 (28.3\%) specimens. Influenza A/H1 N1 pdm09,A/H3 and B viruses isolated in Nepal were antigenically similar to the vaccine strain influenza A/California/07/2009(H1N1pdm09), A/Texas/50/2012(H3N2), A/New York/39/2012(H3N2) and B/Massachusetts/2/2012, respectively.

Conclusions: Influenza viruses were reported year-round in different geographical regions of Nepal which was similar to other tropical countries. The circulating influenza virus type and subtypes of Nepal were similar to vaccine candidate virus which could be prevented by currently used influenza vaccine.

Keywords: Characterization; epidemiology; influenza virus; Nepal.
\end{abstract}

\section{INTRODUCTION}

Influenza viruses, members of the orthomyxoviridae family, circulate worldwide and are a major global health threat. ${ }^{1}$ Influenza viruses cause epidemics of respiratory illness which are often associated with increased hospitalization and mortality. ${ }^{2}$ Intensive research on the molecular evolution of influenza viruses has provided important insights of seasonal genesis and spread in human populations. ${ }^{3}$ They are characterized by genetic and antigenic variability of surface antigens, including haemagglutinin (HA) and neuraminidase (NA) proteins. ${ }^{4}$

Acute respiratory infection remains a global leading cause of death, and influenza is among the most important causes of severe infections and deaths every year. ${ }^{5}$ Current estimates indicate that each year seasonal influenza affects 5 to $10 \%$ of the world's population resulting in 250,000 to 500,000 deaths. ${ }^{6}$ Influenza related complications is often seen in very young, elderly and people with underlying medical conditions. ${ }^{7}$ Therefore, this study was designed to explore molecular epidemiology of seasonal influenza viruses in Nepal.

\section{METHODS}

A descriptive, cross-sectional study was conducted at the National Influenza Center (NIC), National Public Health Laboratory (NPHL), Kathmandu, Nepal from January to December 2014. A total of 1495 throat swab specimens were collected from patients presenting with influenza-like-illness (ILI); which included a fever $>38^{\circ} \mathrm{C}$ in addition to two or more symptoms including cough, running nose, chills and / or sore throat within last seven 
days. These clinical criteria were in accordance with World Health Organization (WHO) case definition for ILI and severe acute respiratory infection (SARI). Specimens were collected and transported to NIC from hospital based sentinel sites of National Influenza Surveillance Network (NISN). Specimens were kept in Viral Transport Media (Copan, Italy), stored at $4^{\circ} \mathrm{C}$ and transported to NIC in cold chain boxes within 24 hours of collection.

Total RNA was extracted using the QIAamp Viral RNA mini kit (QIAGEN GmbH, Hilden, Germany) according to manufacturer's instructions. Real time PCR thermal cycler (Rotor-Gene 6000 Corbett, Australia) was used for typing and subtyping assay of influenza viruses. Real time PCR assay included reverse transcription at $50^{\circ} \mathrm{C}$ for 30 minutes, Taq inhibitor inactivation at $95^{\circ} \mathrm{C}$ for 10 minutes followed by 45 cycles at $95^{\circ} \mathrm{C}$ for 15 seconds, and $55^{\circ} \mathrm{C}$ for 30 seconds. The primers and probes for influenza virus type and subtypes (H1N1, H3N2, H1N1 pdm09, H5N1, and influenza B) were provided by US Center for Disease Control (CDC) and assays were performed according to the protocols. ${ }^{8}$

A monolayer of Madin-Darby Canine Kidney (MDCK) cellline was grown (80-100\%) in T25 flask for isolation of the influenza viruses. Approximately $10 \%$ of specimens tested positive for influenza $\mathrm{A}(\mathrm{H} 1, \mathrm{H} 3$, or $\mathrm{H} 1 \mathrm{~N} 1 \mathrm{pdm} 09)$ and $B$ were inoculated into MDCK cells, incubated at $37^{\circ}$ $\mathrm{C}$ in the presence of $5 \% \mathrm{CO} 2$ for 3-7 days. The flasks showing cytopathic effect (CPE) greater than $80 \%$ of the monolayer cells were harvested. Hemagglutination (HA) test was performed using human ' $\mathrm{O}$ ' group RBC following the WHO standard protocol. ${ }^{9}$ Fifty micro-liter of culture supernatant was added to $U$ shaped micro-well plate containing $50 \mu$ of phosphate buffer solution $(\mathrm{pH} 7.2)$ and a serial two fold dilution was made. The same volume $(50 \mu \mathrm{l})$ of RBC suspension $(0.75 \%)$ was added to all microwells and incubated for one hour at room temperature for a HA reaction. A positive reaction was observed by mat formation in U-shape well of the plates (Greiner, Germany) and settled RBCs in the form of button were recorded negative reactions. The specimens with a HA titer $\geq 1: 32$ were processed for antigenic characterization by hemagglutination inhibition (HI) assay.

A total of 93 viruses were successfully isolated from 148 influenza-positive specimens. A HI assay was used to identify influenza A/H3, A/H1N1 pdm09 and influenza $B$ viruses. Ferret antisera against reference viruses for antigenic characterization of influenza $\mathrm{A} / \mathrm{H} 3, \mathrm{~A} / \mathrm{H} 1 \mathrm{~N} 1$ pdm09 and B viruses were A/Texas/50/2012(H3N2), A/ New York/39/2012(H3N2), A/California/7/2009, and $B /$ Massachusetts/2/2012, respectively similar to WHO recommended composition of influenza virus vaccine for 2014-2015. Also, antigenic characterization of some of A/H3N2 viruses of 2014 were carried out by micro-neutralization (MNT) assays using ferret antisera against reference viruses including vaccine strain of 2014/15 season, A/Texas/50/2012(H3N2) and A/New York/39/2012(H3N2) strain like virus. Hemagglutination inhibition and MNT assays was performed in accordance of WHO manual. ${ }^{9}$ The susceptibility of viruses to four Neuraminidase (NA) inhibitors (Oseltamivir, peramivir, Zanamivir and Laninamivir) were examined by fluorescent NA inhibition assay and expressed as the drug concentration required to inhibit NA activity by $50 \%\left(\mathrm{IC}_{50}\right)$. Antiviral drug sensitivity assay was kindly supported by National Institute of Infectious Diseases, WHO Collaborating Center for Influenza and Research on Influenza, Tokyo, Japan. Nucleotide sequences of $\mathrm{HA}$ gene of influenza viruses were used for phylogenetic analysis. Nucleotide sequences were aligned using the CLUSTALW program. All results were based on pairwise analysis, which was performed using the Maximum Composite Likelihood method in Molecular Evolutionary Genetic Analysis (MEGA) version 5 as described previously. ${ }^{10}$ Each of sequences was registered in Global Initiative on Sharing All Influenza Data (GISAID), a public database (http://www.gisaid.org). Statistical analysis was performed using SPSS-11.5 version, inferential statistic and percentage were generated.

This study was approved by the Nepal Health Research Council (Reg. no. 180/2015).

\section{RESULTS}

A total of 1495 throat swab specimens were received from 59 districts of Nepal; of these influenza virus transmission was found in 36 districts (Figure-1). Influenza viruses were detected in $421(28.2 \%)$ throat swab specimens during the year 2014. The higher number of specimens and positivity of influenza viruses were found in densely populated cities such as Kathmandu (132/432), Lalitpur (129/409), Bhaktapur (26/99) followed by Makawanpur (17/68), Baglung (29/32) and Bhojpur (16/23) district.

Influenza A virus was detected in 301/421 (71.5\%) specimens; of which $120(39.9 \%)$ were influenza A /H1N1 pdm09 and $181(60.1 \%)$ were influenza A/H3 subtype. Similarly, influenza B virus was detected in 119 (28.3\%) and $1(0.2 \%)$ specimen was found influenza $\mathrm{A} / \mathrm{H} 3$ and influenza B co- infection. Among the total cases, 1078 (72.1\%) had an ILI and 417 (27.9\%) had shown SARI like clinical presentation. Of those with an ILI, 369 (34.2\%) were influenza positive and of those with SARI, 52 (12.5\%) specimens were positive for influenza virus (Table-1). 


\section{Table 1. Influenza types and subtypes among ILI \& SARI cases, 2014.}

\begin{tabular}{|llllll|}
\hline Case & \multicolumn{2}{c}{ Influenza A (\%) positive } & Influenza B (\%) positive & Negative & Total Cases \\
& A/H1N1pdm09 & Influenza A/H3 & & & \\
ILI & $101(9.36)$ & $164(15.21)$ & $104(9.64)$ & $309(65.76)$ & 1078 \\
\hline SARI & $19(4.79)$ & $18(4.31)$ & $15(3.59)$ & $165(87.5)$ & 417 \\
Total & 120 & 182 & 119 & 1074 & 1495 \\
\hline
\end{tabular}

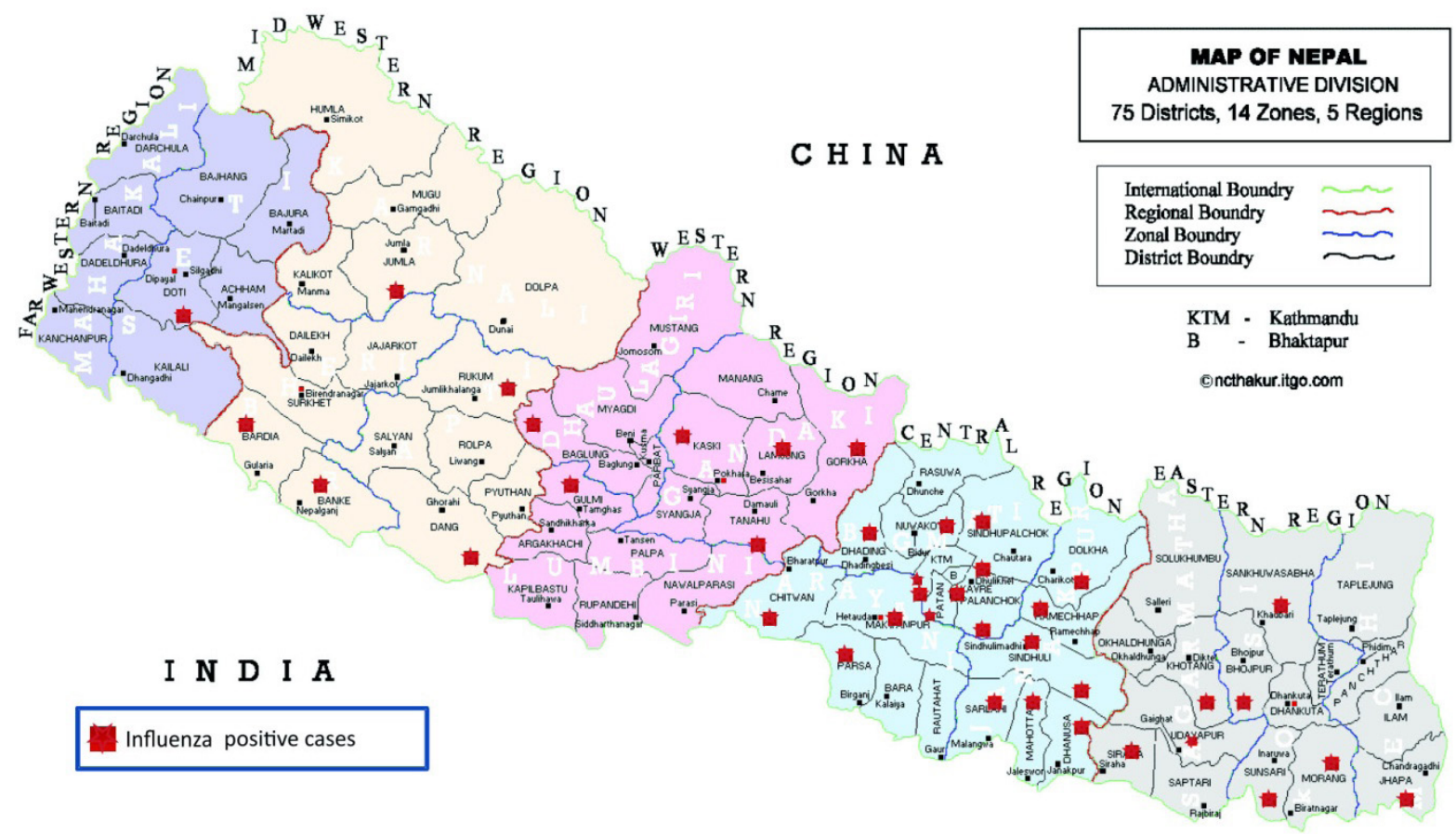

\section{Figure1. District wise transmission of influenza viruses, 2014.}

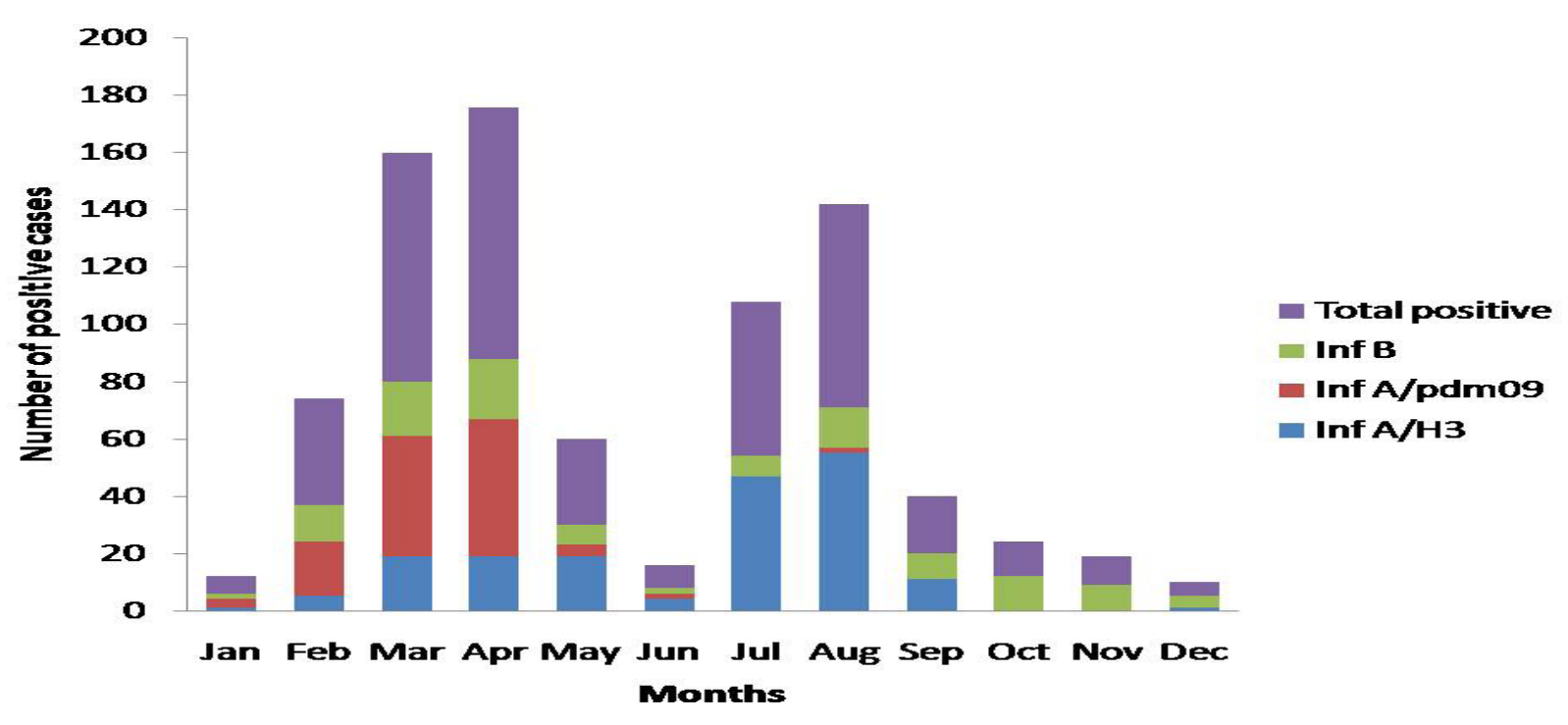

Figure 2. Month-wise distribution of influenza virus types and subtypes, 2014.

Influenza cases were found throughout the year 2014 with the highest number of cases reported in April (21\%), March (19\%) and August (16.8\%) followed by July
(12.8\%) and February (8.8\%). The lowest number of cases was seen during December (1.2\%). Influenza A/H3 was detected year-round except October and November. 
Similarly, influenza A/H1N1 pdm09 was not detected in between September to December and also in July. Influenza B was reported year round, being highest in April (Figure-2).

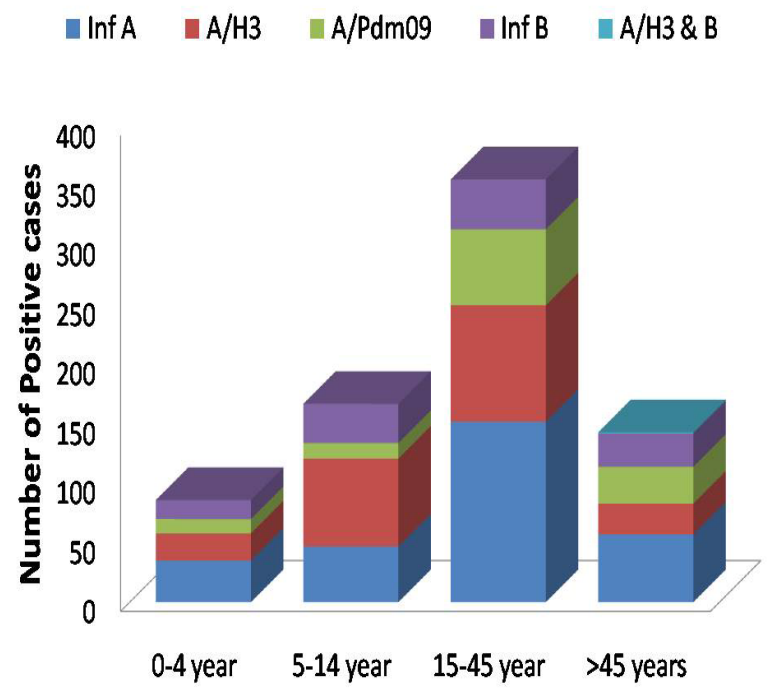

Figure 3. Age group-wise distribution of influenza type and subtypes, 2014

Among influenza $A$, the number of influenza $A / H 3$ (15.5\%), was highest in age group 15-45 years followed by $5-14,>45$ and $0-4$ years respectively. Similarly, influenza $B(6.6 \%)$ was highest in age group $15-45$ years followed by $5-14,>45$ and $0-4$ years old respectively. The positive cases of influenza A/H1N1 pdm09 (10.1\%) were higher among 15-45 years followed by $>45,5-14$ and 0-4 years (Figure-3). These results demonstrated that influenza viruses could lead to substantial burden on health especially in between 15 to 45 year and elderly populations of Nepal.

By $\mathrm{HI}$ assay, we found that influenza A / H1N1 pdm09, $\mathrm{A} / \mathrm{H} 3$ and $\mathrm{B}$ viruses of Nepal were antigenically similar to the influenza A/California/07/2009, A/ Texas/50/2012(H3N2), A/New York/39/2012(H3N2), $B /$ Massachusetts/2/2012 virus, respectively. The phylogenetic tree of HA gene of influenza A/H1N1 pdm09 viruses can be divided into 7 genetic clades. Recent influenza A/H1N1 pdm09 viruses belonged to clade 6 which was circulated worldwide during influenza seasons in 2014. Phylogenic analysis of influenza A/H1N1 pdm09 viruses revealed subclade 6B. A total of influenza A/H1N1 pdm09virus $(n=6)$ of the year 2013 was isolated during the year 2014 were clade 6C (Figure. 4). Phylogenetic analysis based concatenation studies of viral genomes had identified seven genetic clades worldwide and clade eight restricted to the African west. ${ }^{11}$ Circulation of clade 6 and 7 prevailed worldwide and similar findings were published in Brazil, Hong Kong, India, Japan and the United States. ${ }^{12}$

Phylogenetic analysis of influenza $A(H 1 N 1)$ pdmog HA genes

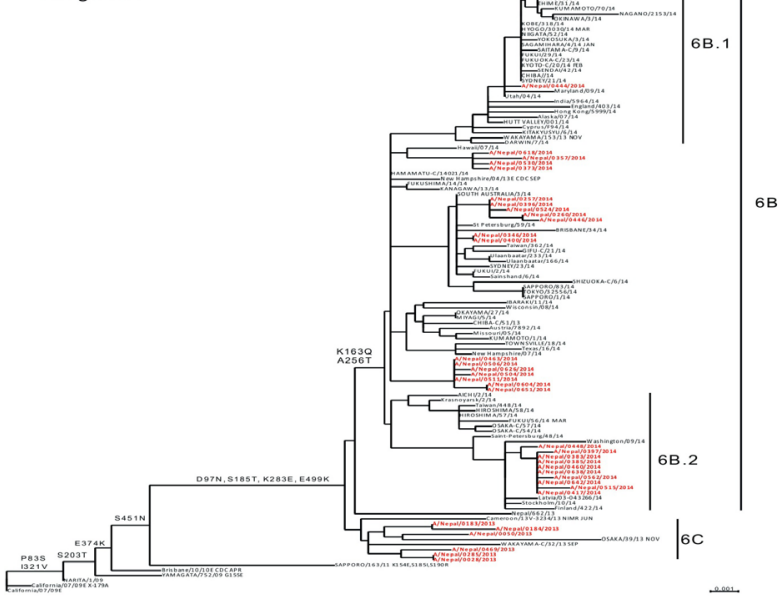

Figure 4. The phylogenetic analysis of HA gene of influenza A/H1N1 pdm09 viruses of Nepal with reference strains. The tree was constructed using the Neighbor-Joining method using mega software version 5 .

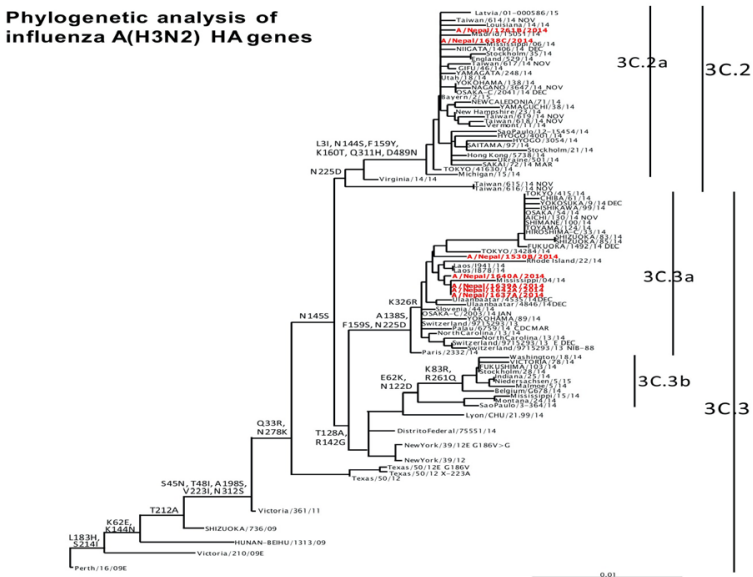

Figure 5. Phylogenetic analysis of HA gene of influenza A/H3N2 viruses of Nepal with reference strains. The tree was constructed using the Neighbor-Joining method using mega software version 5.

Antigenic analysis of some $\mathrm{A} / \mathrm{H} 3$ viruses was carried out by microneutralization test (MNT). All viruses tested by MNT, except for influenza A/Nepal/1640A/2014 showed a reduction in reactivity with ferret antisera against MDCK-SIAT1 cell-grown A/New York/39/2012 (H3N2) virus (data not shown). Influenza A/Nepal/1640A/2014 was antigenically similar to MDCK-SIAT1 cell-grown A/ 
New York/39/2012 (H3N2). These viruses belonging to subclade 3C.2a and 3C.3a (Figure. 5) possessing mutations in antigenic sites in the HA gene at amino acid positions N144S, F159Y, K160T (3C.2a), A138S and F159S (3C.3a) respectively. In our study, rests of all the viruses were sensitive to oseltamivir, peramivir, zanamivir and laninamivir.

\section{DISCUSSION}

Nucleic acid amplification tests are the preferred methods for identification of respiratory viral infections, including influenza and PCR-based methods provide rapid, sensitive detection and most importantly, helpful with identifying different subtypes of influenza viruses. ${ }^{13}$

Influenza Like Illness was found year-round in Nepal with a predominant A/H3 subtype in 2013 and 2014. Influenza cases were highest during August, July, March followed by February and April. Higher numbers of ILI cases were reported from Kathmandu, Lalitpur, Bhaktapur, Makawanpur, Baglung and Bhojpur districts which have a distinct geography and climatic variation. Similar findings were reported from Srinagar (JanuaryMarch), Delhi (July-September), Lucknow (June- July), Kolkata (June-July) and Pune (July-September) of India where subtype A/H3 was predominant in 2011, 2012 and $2013 .{ }^{14}$ Similarly, the predominant subtype in most tropical South America was influenza A/H3N2, with cocirculation of influenza A/H1N1 pdm09 and influenza B. ${ }^{15}$

Human influenza transmission usually occurs in the winter season in the northern hemisphere temperate region but the exact timing and duration of the influenza season varies by country and year. ${ }^{16}$ Many studies investigated the seasonal patterns of influenza but the exact mechanisms of spread and emergence of new variant strains of viruses are still not well understood. ${ }^{1}$

From the public health prospective, information on seasonality of pathogens is crucial to inform the timing of interventions, particularly for a climatically and economically diverse country. ${ }^{17}$ In this perspective, effective influenza surveillance systems are essential to understand the epidemiology and seasonality of influenza and for optimizing influenza control strategies. ${ }^{14}$

Annual seasonal influenza epidemics alone causes significant morbidity and mortality, affecting 5-15\% of the global population, hence are of major public health concern. ${ }^{18}$ The study conducted in Thailand, ${ }^{19}$ Singapore, ${ }^{20}$ Vietnam, ${ }^{21}$ Philippines ${ }^{22}$ have shown that there is a substantial burden of influenza in South-East Asia. ${ }^{23}$ In Nepal, the number of ILI cases reached at peak (28.2\%) in 2014 which is similar to other countries belonging to South-East Asia.

Influenza H3N2 subtype was predominant during 2014 in Nepal and a similar finding was reported from the European countries dominated by influenza A/H3N2 although, both $\mathrm{A} / \mathrm{H} 1 \mathrm{~N} 1 \mathrm{pdm09}$ and $\mathrm{B}$ viruses were cocirculated. ${ }^{24}$ Of note, influenza $\mathrm{B}$ activity was observed year-round, being highest in February. South East Asia, including Cambodia, Laos People's Republic, The Philippines, Thailand and Singapore reported overall decreasing influenza activity, with influenza A/H3N2 viruses predominating during the year 2014. ${ }^{25}$ The findings of our study could be helpful to recommend vaccine selection and in preparedness for the influenza outbreak. All of the viruses to four NA inhibitors (oseltamivir, peramivir, zanamivir and laninamivir) were found to be sensitive. In a similar study conducted in Beijing, China, all of the A/H1N1 pdm09 viruses isolated in 2012-2013 were sensitive to oseltamivir. ${ }^{26}$

Circulating strains of the virus during influenza season in Nepal revealed many similarities with our neighboring countries and the region. Our finding showed year-round transmission with a peak influenza activity during the rainy and winter season is similar to Thailand, Northern Vietnam and Lao-PDR. Factors driving seasonality of transmission are not well defined but likely include a combination of climatic conditions, susceptibility of the population, and virus characteristics. ${ }^{6}$

This study had various constrains, such as limited number of specimens were analyzed which may not be representative of entire population of affected districts. Because of limited funds and resources, we could not perform detailed genetic characterizations. However, effective and continued influenza surveillance systems are essential to understand the epidemiology and seasonality of influenza and for optimizing control strategies.

\section{CONCLUSIONS}

Circulation of seasonal influenza viruses was found in various geographical regions throughout the year which were similar to other tropical and sub-tropical countries in South-East Asia.Influenza viruses isolated in Nepal are similar with vaccine candidate virus which could be prevented by currently used influenza vaccine.

\section{ACKNOWLEDGEMENTS}

The authors are thankful to all health care providers and laboratory staffs working at national influenza network. We also express gratitude to Shinji Watanabe, Takato 
Odagiri; WHO Collaborating Centre for Reference and Research on Influenza at National Institute of Infectious Diseases, Japan for genetic characterization and antiviral drug susceptibility assay; Centers for Disease Control and Prevention (US, CDC) for support of real time PCR kits and Central Department of Microbiology, Kirtipur. Similarly, we also acknowledge the authors, originating and submitting laboratories of the sequences from GISAID's EpiFlu'M ${ }^{\top M}$ Database on which phylogenetic tree were constructed and analyzed. We are thankful to Kedar Baral, Patan Academy of Health Sciences for manuscript review and feedback. The authors are thankful to Mr. Bimlesh Kumar Jha, Ms. Alisha Sapkota, Mr. Tribhuvan Prasad Shah, Ms. Suni Dangol, Mr. Uday Yadav for their support in processing, isolation and identification of influenza viruses at National Influenza Center, Nepal.

\section{REFERENCES}

1. Rutvisuttinunt W, Chinnawirotpisan P, Thaisomboonsuk B, Rodpradit P, Ajariyakhajorn C, Manasatienkij W, et al. Viral subpopulation diversity in influenza virus isolates compared to clinical specimens. J ClinVirol. 2015;86:1623. [PubMed]

2. Ikawati HD, Setiawaty V. Epidemiology of Influenza-like Illness (ILI) in Java Island, Indonesia in 2011. Public Health Research. 2014;4(4):111-6.

3. Nelson MI, Edelman L, Spiro DJ, Boyne AR, Bera J, Halpin R, et al. Molecular epidemiology of A/H3N2 and A/H1N1 Influenza Virus during a single epidemic season in the United States. PLoS Pathog. 2008; 4(8): e1000133. [PubMed]

4. Fang Q, Gao Y, Chen M, et al. Molecular epidemiology and evolution of A (H1N1) pdm09 and H3N2 virus during winter 2012-2013 in Beijing, China. Infect Genet Evol. 2014;26:228-40. [PubMed]

5. $\mathrm{Ng} \mathrm{S}$, Gordon A. Influenza burden and transmission in the tropics. Curr Epidemiol Rep. 2015;2 (2):89-100. [PubMed]

6. World Health Organization. A manual for estimating disease burden associated with seasonal influenza. Geneva:WHO; 2015.

7. Nukiwa N, Burmaa A, Kamigaki T, Darmaa B, Od J, Od I, et al. Evaluating influenza disease burden during the 2008-2009 and 2009-2010 influenza seasons in Mongolia. Western Pac Surveill Response J.2011;2(1):16-

\section{2. [PubMed]}

8. CDC protocol of real-time RTPCR for swine influenza A (H1N1)- WHO. 28 April 2009, 261 revision-1 30 April 2009. Available at: http://www.who.int/csr/resources/ publications/swineflu/CDCrealtimeRTPCRprotocol_20 090428.pdf

9. Manual for the laboratory diagnosis and virological surveillance of influenza WHO. 2011, Available at: http://whqlibdoc.who.int/ publications / 2011/9789241548090_eng.pdf

10. Tamura K, Dudley J, Nei M, Kumar S. MEGA4: Molecular Evolutionary Genetis Analysis (MEGA) software version 4.0. Mol Biol Evol. 2007;24 (8):1596-9. [PubMed]

11. Klimov AI, Garten R, Russell C, Barr IG, Besselaar TG, Daniels R, et al. WHO recommendations for the viruses to be used in the 2012 Southern Hemisphere influenza vaccine: epidemiology, antigenic and genetic characteristics of influenza $\mathrm{A}(\mathrm{H} 1 \mathrm{~N} 1)$ pdm09, $\mathrm{A}(\mathrm{H} 3 \mathrm{~N} 2)$ and B influenza viruses collected from February to September 2011. Vaccine. 2012; 30 (45): 6461-71. [PubMed]

12. Arencibia A, Acosta B, Muné M, Valdés $O$, Fernandez L, Medina I, et al. New genetic variants of influenza A(H1H1) pdm09 detected in Cuba during 2011-2013. Infect Genet Evol. 2015;(32):322-6. [PubMed]

13. ChoiYJ, Nam HS, Park JS, Kim HJ, Park KB, Jeon MH, et al. Comparative analysis of the multiple test methods for the detection on pandemic influenza A/H1N1 2009 virus. J Microbial Biotechnol. 2010;20(10):1450-6. [PubMed]

14. Chadha MS, PotdarVA, Saha S, Koul PA, Broor S, Dar L, et al. Dynamics of influenza seasonality at sub-regional levels in India and implications for vaccination timing. PLoS ONE. 2015;10(5):e0124122. [PubMed]

15. World Health Organization. Weekly epidemiological record. 2014;48(89): 529-44. Available at: http://www. who.int/wer/2014/wer8948.pdf

16. Hammond A, Gusbi N, Sosa P, Fitzner J, Besselaar T, Vandemaele K, et al. Review of the 2014-2015 influenza season in the northern hemisphere. Wkly Epidemiol Rec. 2015;23(90):281-96. Available at: http://www.who.int/ wer/2015/wer9023.pdf

17. Feng L, Li Z, Zhao S, Nair H, Lai S, Xu W, et al. Viral etiologies of hospitalized acute lower respiratory infection patient in China, 2009-2013. PLoS One. June 19,2014;9(6):e99419. [PubMed]

18. Dangi T, Jain B, Singh AK, Mohan M, Dwivedi M, Singh JV, et al. Influenza virus genotypes circulating in and around 
Lucknow, Uttar Pradesh, India, during post pandemic period, August 2010-September 2012. Indian J Med Res. 2012:139(3):418-26. [PubMed]

19. Simmerman JM, Chittaganpitch M, Levy J, Chantra S, Maloney S, Uyeki T, et al. Incidence, seasonality and mortality associated with influenza pneumonia in Thailand, 2005-2008. PLoS One. 2009;4(11):e7776. [PubMed]

20. Ang LW, Lim C, Lee VJM, Ma S, Tiong WW, Ooi PL, et al. Influenza-associated hospitalizations, Singapore, 2004-2008 and 2010-2012. Emerg Infect Dis. 2014;20(10):1652-60. [PubMed]

21. Le MN, Yoshida LM, Suzuki M, Nguyen HA, Le HT, Moriuchi H, et al. Impact of 2009 pandemic influenza among Vietnamese children based on a population-based prospective surveillance from 2007 to 2011. Influenza Other Respir Viruses. 2014;8(4):389-96. [PubMed]

22. Tallo VL, Kamigaki T, Tan AG, Pamaran RR, Alday PP, Mercado ES, et al. Estimating influenza outpatients' and inpatients' incidences from 2009 to 2011 in a tropical urban setting in the Philippines. Influenza Other Respir Viruses. 2014;8(2):159-68. [PubMed]
23. Sam JI. The burden of human influenza in Malaysia. Med J Malaysia. 2015;70(3):127-30. [PubMed]

24. Broberg E, Snacken R, Adlhoch C, Beauté J, Galinska M, Pereyaslov D, et al. Start of the 2014/15 influenza season in Europe: drifted influenza A(H3N2) viruses circulate as dominant subtype. Euro Surveill. 2015;20(4):pii:21023. [PubMed]

25. World Health Organization. Influenza updates N-246, 21 September 2015. Available at: http:// www.who.int/influenza/surveillance_monitoring/ updates/2015_09_21_surveillance_update_246.pdf

26. Khodadad N, Moattari A, Shahr-Abadi MS, Kadivar MR, Sarvari J, Tavakoli F, et al. Prevalence of influenza A(H1N1) pdm09 virus resistant to Oseltamivir in Shiraz, Iran, during 2012-2013. Jundishapur J Microbiol. 2015;8(8):e23690. [PubMed] 